\title{
HEBERLÉ S.A.
}

FOURNITURES INDUSTRIELLES EN GROS

GENEVE

Rue du Rhone 61 et Grand-qual 42 Telephone 41377 (2 lignes)

Amiante

Bottes en caoutchouc

Chiffons d'essuyages blancs et couleurs

Courroies de transmissions caoutchouc, cuir ou tissées

Déchets de coton d'essuyage blancs et couleurs

Eponges

Eponges naturelles et viscose

Feutre industriel

Joints en tous genres

Peaux de daim

Produits abrasifs "SIA "

Tabliers industriels en caoutchouc, cuir, GURIT, toile

Tapis en caoutchouc

Tuyaux d'arrosage, à air comprimé, à vin, à vapeur, pour radiateurs

Tous tuyaux caoutchouc pour lindustrie

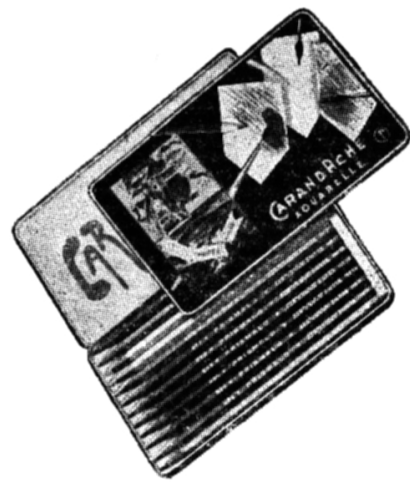

PRISMALO 999

Le meillew crazean de coubow

hour picole

\section{PAPETERIE DE BALSTHAL}

BALSTHAL (Solouro)

OUATE DE CELLULOSE

PAPIER CREPPE POUR PANSEMENTS

LINGERIE EN PAPIER

Bureau de vente :

BAREISN, WIELAND \& C $C^{\text {10 }}$ s. A. Kornhausbrücke, 7 - ZURICH - Téléphone 234724 


\section{LA BALDISE}

COMPAGNIE D'A SSURANCES contre les risques de transport

BALE (Suisse)

\section{Fondée en 1864}

La maison de confiance qui est spécialisée pour les assurances maritimes, fluviales, terrestres et aériennes.

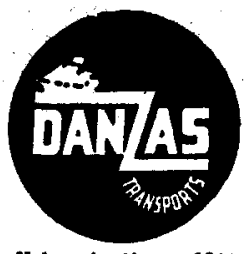

\section{TRAMSPORTS IMTERMATIOMAUX}

\section{PASSAGES - VOYAGES}

Malsen lendes on 1815

Socisté par Aotions

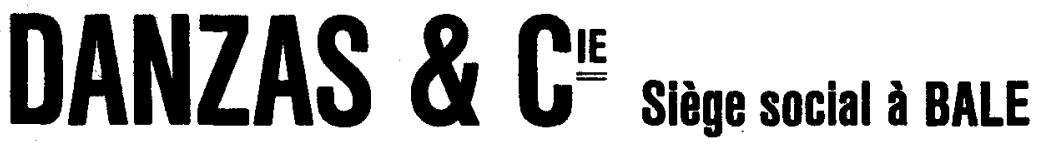

Succursales a GNEVE, Gare Voyageurs-Cornavin, Brigue, Buchs, Chlasso, Lucerne, St-Margrethen, Schaffhouse, St-Gall, Vallorbe, Zurich, Parls, 15, rue de Nancy $\left(10^{\circ}\right)$, Belfort, Nancy, Annemasse, Bellegarde, Besançon. Delle, Pontarller, Modane.

Bureaux de voyages à GENEVE, rue du Mont-Blanc No 5, Bale, Buchs, Lugano, Schaffhouse, St-Gall, Zurich. 


\section{Institul Minepva Zurich Préparation à Section commerclalo \\ I'Université el Cours pour \\ l'Ecole Polytechnique aldes-médecins}

\section{UNIVERSITÉ \\ DE LAUSANNE}

Facultés de Théologie, Droit, Médecine, Lettres et Sciences.

Ecoles de sciences sociales et politiques, hautes études commerciales, pharmacie.

Institut de police scientifique.

\section{ÉCOLE POLYTECHNIQUE ÉCOLE oB FRANÇAIS MODERNE}

RENSEIGNEMENTS

AU SECRÉTARIAT DE L'UNIVERSITE

\section{BUFFETS}

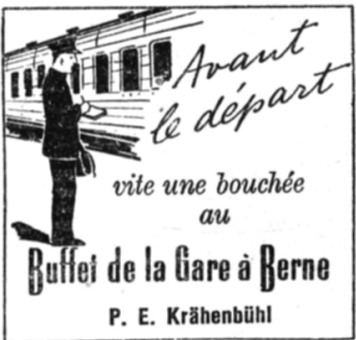

\section{HOTELS}

La Résidence, Genève

11, Florissant

Tél. 4 I 388

\section{Hôtel-Restaurant - Bar}

150 chambres avec téléphone

60 salles de bains

Tennis - Parc pour autos

Arrangements pour familles

H O T E L

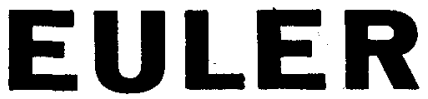

Ier RANG

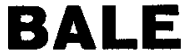

En face de la Gare CFF

Téléphone: Télégramme :

21723 Eulerhotel

\section{CLINIQUES}

La Clinique MONT-RIANT à Chamby sur Montreux

Spécialement organisée pour

les cures de repos, les convalescences et le traitement

des maladies chroniques vous offre

des chambres avec loggias, des chambres au soleil avec et sans balcons, des appartements avec salle de bain, une cuisine soignée et une situation privilégiée au prix de Fr. 14, - a 28,- par jour. (Arrangements pour longs séjour.)

Dr GOD A R D, médecin-directeur 


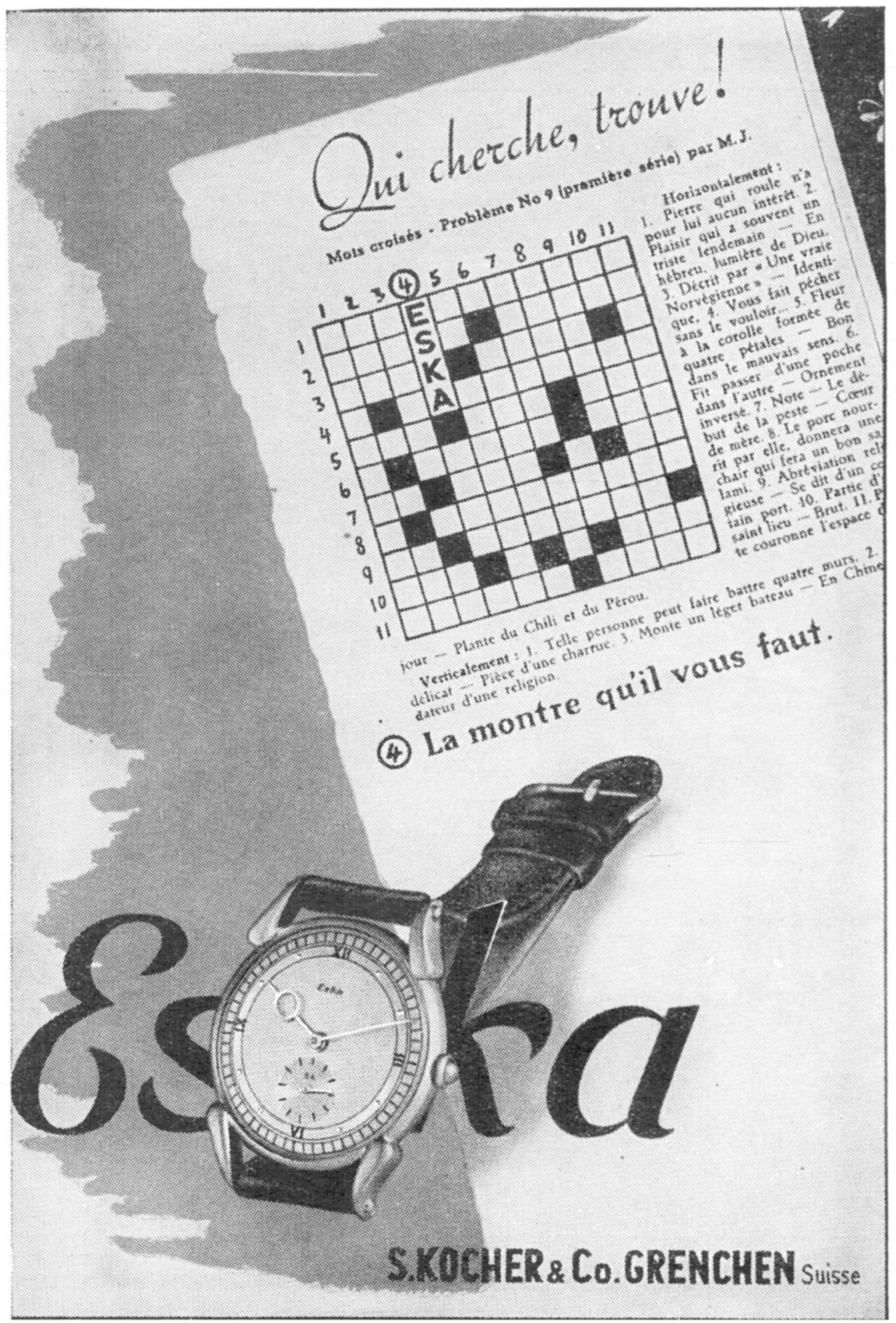




\section{ADRESSES DES COMITES CENTRAUX DES SOCIETÉS NATIONALES DE LA CROIX-ROUGE}

ALBANIE - Croix-Rouge albanaise, Tirana.

ALLEMAGNE - Les groupements régionaux de la CroixRouge ont formé des Comités de coordination dans la zone américaine (Junghofstrasse 20, Francfort/M.), dans la zone britannique (Harvestehuderweg 26, Hambourg 13) et dans la zone française (Maximilianstr. 1, Baden-Baden). Il existe aussi des groupements régionaux dans la Sarre et dans la zone du Grand-Berlin.

ARGENTINE - Croix-Rouge argentine, Hipolito Irigoyen 2008, Buenos-Aires.

AUSTRALIE - Croix-Rouge australienne, 122-128, Flinders Street, Melbowrme.

AUTRICHE - Crolx-Rouge autrichienne, Milchgasse 1 V'ienne I

BELGIQUE - Croix-Rouge de Belgique, 98, Chaussée de Vleurgat, Bruxelles.

BIRMANIE - Croix-Rouge de Birmanie, Office of the director of public Health, Phayre Street, Rangoon.

BOLIVIE - Croix-Rouge holivienne, Casilla num. 741, Lu Pas.

BRÉSIL - Croix-Rouge brésillenne, Praça de Cruz Vermelha, 10-12, Rio de Janeiro.

BULGARIE - Croix-Rouge bulgare, 21, boul. Totleben, Sofia.

CANADA - Croix-Rouge canadienne, 95, Wellesley Street, Toronto, 5 .

CHILI - Croix-Rouge chilienne, Calle Catedral, 1572, Correo, 15, Casilla, 246, V., Santiago de Chile.

CHINE - Croix-Rouge chinoise, 275, Chungsan Lu Road, Nankin.

COLOMBIE - Croix-Rouge colombienne, Calle 8a, num. 7-73. Apartado, nacional 1110, Bogota.

COSTA-RICA - Croix-Rouge costaricienne, Calle 5a Sur, San José.

CUBA - Croix-Rouge cubaine, Zulueta, 461. La Havane.

DANEMARK - Croix-Rouge danoise, Amaliegade, 18, Copenhague $R$.

REPUBLIQUE DOMINICAINE - Croix-Rouge dominicaine Calle Galvan, 24, Apartado 1288, Cuidad Trujillo.

ÉGYPTE - Société nationale Fouad Ier du Croissant-Rouge égyptien, rue Malika Nazli, 34, Le Caire,

EQUATEUR - Croix-Rouge équatorienne. Avenida Colombia y Elizalde, 118, Quito.

ESPAGNE - Croix-Rouge espagnole, Eduardo Dato, 18, Madrid.

ÉTATS-UNIS - Croix-Rouge américaine. National Headquarters, 17th and D. Streets, N.W., Washington 13, D.C.

ÉTHIOPIE - Croix-Rouge éthiopienne, Addis-Abéba.

FINLANDE - Croix-Rouge de Finlande, Tehtaankatu I A, Helsinki.

FRANCE - Croix-Rouge française, 17, rue Quentin-Bauchart, Paris ( $\left.8^{\mathrm{me}}\right)$.

GRANDE-BRETAGNE - Croix-Rouge britannique, 14, Grosvenor Crescent, Londres S.W.1.

GRECE - Croix-Rouge hellénique, rue Mackenzie-King, 1 et rue Solonos, 39, Athines.

GUATÉMALA - Croix-Rouge du Guatémala, 4a, CalleOriente, 64, Guatemala.

HAITTI - Croix-Rouge haĩtienne, rue Férou, Port-au-Prince. HONDURAS - Croix-Rouge du Honduras, Tegucigalpa. HONGRIE - Croix-Rouge hongroise, Baross utca, 15, Budapest VIII.

INDE - Croix-Rouge de l'Inde, no 20, Talkatora Road, New Delhi, 8.
IRAK - Croissant-Rouge de l'Irak, Bagdad.

IRAN - Société du Lion et Soleil Rouges de l'Iran, Avenu Argue, Téhéran.

IRLANDE - Croix-Rouge irlandaise, 25 Westland Row, Dublin

ISLANDE - Croix-Rouge islandaise, Thorvaldsensstraeti, 6 Reykjavik.

ITALIE - Croix-Rouge italienne, 12, via Toscana, Rome.

JAPON - Croix-Rouge japonaise, 5, Shiba Park, Tokio.

JORDANIE - Croissant-Rouge jordanien. A mman.

LIBAN - Croix-Rouge libanaise, Beyrouth.

LIECHTENSTEIN - Croix-Rouge du Liechtenstein, Vadus

LUXEMBOURG - Croix-Rouge luxembourgeoise, 5, rue d Rollingergrund, Luxcmbourg.

MEXIQUE - Croix-Rouge mexicaine, Esq. Durango Montertey Col. Roma, Mexico DF.

MONACO - Croix-Rouge monégasque, Monaco.

NICARAGUA-Croix-Rouge du Nicaragua, Managua D.N.C.A

NORVEGE - Croix-Rouge de Norvège, Bankplassen, 1, Osk

NOUVELLE-Z ELANDE - Croix-Rouge néo-zélandaise 61, Dixon Street, Wellinglon C.1.

PAKISTAN - Croix-Rouge du Pakistan, Katrak Building Mansfield Road, Karachi $I I I$.

PANAMA - Croix-Rouge de Panama, Panama.

PARAGUAY - Croix-Rouge paraguayenne, Avenida Espane 505, Asuncion.

PAYS-BAS - Croix-Rouge néerlandaise, 27, Princessegrach1 La Haye.

PÉROU - Croix-Rouge péruvienne, Avenida Nicolas d Piérola, 224, Lima.

PHILIPPINES - Croix-Rouge philippine, 166, San Raphae Street, Manille.

POLOGNE - Croix-Ruuge polonaise, Mokotowska, 14 Varsovic.

PORTUGAL - Croix-Rouge portugaise, Secrétariat généra Praça do Comércio, 1, Lisbonne.

ROUMANIE - Croix-Rouge roumaine, Strada Biseric Amzei, 29, Bucarest.

SALVADOR - Croix-Rouge du Salvador, 3a, Calle Poniente 21, San Salvador.

SIAM - Croix-Rouge siamoise, King Chulalongkorn Memoria Hospital, Bangkok.

SUEDE - Croix-Rouge suédoise, Artillerigatan, 6, Stockholm 14

SUISSE - Croix-Rouge suisse, Taubenstrasse, 8, Berne.

SYRIE - Croissant-Rouge syrien, Damas.

TCHECOSLOVAQUIE - Croix-Rouge tchécoslovaqui Thunovska, 18, Prague $I I I$.

TURQUIE - Société du Croissant-Rouge turc, Yenisehir Ankara.

UNION SUD-AFRICAINE - Croix-Rouge sud-africain His Majesty's Building, Floff Street. P.O. Box 8728 Johannesburg.

U.R.S.S. - Alliance des Sociétés de la Croix-Rouge ot di Croissant-Rouge de l'U.R.S.S., rue Neglinnaya, 25, Moscow

URUGUAY - Croix-Rouge uruguayenne, Avenida, 8 de Octubre, 2990, Montevideo.

VENEZUELA - Croix-Rouge vénézuélienne, Avenida Este 303-3, Caracas.

YOUGOSLAVIE - Croix-Rouge yougoslave, Simina ulic: broj, 19, Belgrade. 